\title{
Eficacia de la Azitromicina asociada al raspado y alisado radicular en periodontitis crónica
}

\section{Efficacy of azithromycin associated with scaling and root planing in chronic periodontitis}

\author{
Pablo Millones-Gómez¹ , Alberto Aguirre Aguilar²
}

\begin{abstract}
1. Departamento de Cirugía Bucal y MaxilofacialUniversidad Católica Los Ángeles de Chimbote - Trujillo- Perú

2. Departamento de Periodoncia- Universidad

Nacional de Trujillo- Trujillo- Perú
\end{abstract}

Correspondencia a: Pablo Alejandro Millones Gómez | Teléfono: 51949371052 | Email: pablodent@hotmail.com | Trujillo-Perú Trabajo recibido el 11/07/2016. Aprobado para su publicación el 11/12/2016

\section{RESUMEN}

Objetivo: evaluar la eficacia de la azitromicina asociada al RAR (asociada al raspado y alisado radicular) en pacientes con periodontitis crónica. Material y método: El presente ensayo clínico paralelo aleatorizado, controlado y triple ciego, se desarrolló en la Clínica Estomatológica de la Universidad Católica Los Ángeles de Chimbote (Trujillo, Perú), entre marzo y diciembre de 2015. Participaron 30 pacientes adultos, distribuidos aleatoriamente en 2 grupos, designados con las letras A y B, de 15 integrantes cada uno. Cada paciente fue evaluado periodontalmente antes de realizarle el raspado y alisado radicular y optimización de las técnicas de higiene. A los pacientes del grupo A se les indicó tratamiento antibiótico con Azitromicina 500 mg por vía oral cada 24 horas durante 3 días y al grupo $B$ se les indicó un placebo con las mismas características físicas del antibiótico escogido. Los pacientes fueron evaluados a los 3,4 y 5 meses. La eficacia fue evaluada mediante el test $\mathrm{T}$ de Student para comparación de medias, considerando un nivel de significancia para los valores de $p<0,05$. Resultados: Se encontró que ambos grupos lograron mejorar la profundidad de sondaje y el nivel de inserción clínica sin embargo no presentaron diferencias estadísticamente significativas entre ellos. Conclusión: La azitromicina asociada al RAR no es eficaz como terapia coadyuvante en pacientes con periodontitis crónica.

PALABRAS CLAVE:

Azitromicina, raspado y alisado radicular, periodontitis crónica

Rev. Clin. Periodoncia Implantol. Rehabil. Oral Vol. 10(1); 33-37, 2017.

\section{ABSTRACT}

Objective: To evaluate the efficacy of azithromycin associated with the RAR (Associated with scaling and root planing) in patients with chronic periodontitis. Material and Methods: This clinical trial parallel randomized, controlled, triple blind, was developed in the Stomatology Clinic of the Catholic University Los Angeles of Chimbote (Trujillo, Peru), between March and December 2015. The participants were 30 adult patients randomized 2 groups, designated by the letters $A$ and $B$ of 15 members each. Each patient was evaluated periodontally before Give you the scaling and root planing and optimization hygiene techniques. Patients in group A, were prescribed antibiotic treatment with azithromycin $500 \mathrm{mg}$ orally every 24 hours for 3 days. While, group B were prescribed a placebo with the same physical characteristics of the antibiotic chosen. Patients were evaluated at 3,4 and 5 months. The efficacy was evaluated using the Student $t$ test to compare means, considering a level of significance for the values of $p<0.05$. Results: It was found that both groups were able to improve probing depth and clinical attachment level however no statistically significant differences between them. Conclusion: Azithromycin associated with the RAR is not effective as adjunctive therapy in patients with chronic periodontitis.

\section{KEY WORDS:}

Azithromycin, scaling and root planing, chronic periodontitis

Rev. Clin. Periodoncia Implantol. Rehabil. Oral Vol. 10(1); 33-37, 2017. 


\section{INTRODUCCIÓN}

La periodontitis crónica es una infección mixta causada principalmente por bacterias periodontopatógenas que residen en la placa subgingival. El primer paso en su tratamiento es reducir la infección, inflamación y bacterias causantes mediante el raspado y alisado radicular. ${ }^{1,2}$

El reconocimiento del origen y especificidad de los microorganismos en la enfermedad periodontal fueron identificadas al finalizar la década de los 70 del siglo pasado, incrementándose el interés por el empleo de terapias antimicrobianas que promuevan la supresión de los probables agentes etiológicos. A partir de esa fecha muchos grupos de investigadores empezaron a investigar los efectos de la administración sistémica de estos antimicrobianos como coadyuvantes al raspado y alisado radicular. Desafortunadamente por muchos años los resultados de esas investigaciones han sido inconclusas o contradictorias, especialmente al medir los efectos sobre la composición de la flora subgingival. ${ }^{2}$

Las dificultades técnicas para evaluar el complejo microbiano subgingival que es altamente colonizado por microorganismos anaerobios estrictos pasa por las carencias de métodos microbiológicos adecuados, sin embargo, al descubrir técnicas que no son dependientes de la viabilidad de la bacteria para su identificación como PCR, microarrays, u oligonucleótidos se lograron conocer los agentes etiológicos de la enfermedad periodontal. ${ }^{3}$

Los resultados de estos estudios confirman el rol de ciertos microorganismos como: Aggregatibacter actinomycetemcomitans, Porphyromonas gingivalis, Treponema denticola, Tannerella forsythia, Prevotella intermedia, Parvimonas micra, Fusobacterium nucleatum, Selenomonas sputigena and Eubacterium nodatum en la aparición y el avance de la periodontitis. ${ }^{4} \mathrm{Y}$ tras la inclusión de pruebas de diagnóstico molecular incluyendo métodos de secuenciación se logró identificar a otros microorganismos que se encuentran presente y podrían ser especies patógenas asociadas a la aparición de enfermedad periodontal, dentro de estas nuevas especies encontramos a: Porphyromonas endodontalis and Bacteroidales y dentro de las spiroquetas: Treponema lecithinolyticum y Treponema médium; Firmicutes: Filifactor alocis y S. sputigena. . $^{4}$

Estos hallazgos han desempeñado un papel importante en nuestra comprensión de la etiología de las enfermedades periodontales, especialmente periodontitis crónica. Además, los resultados de estos estudios sugieren que con el fin de obtener efectos terapéuticos exitosos, es tan importante fomentar el crecimiento de especies bacterianas compatible con el huésped como lo es para eliminar o suprimir los patógenos periodontales.

Los criterios de valoración clínica para la terapia periodontal son muy sencillos e incluyen ganancia de inserción clínica, la reducción de la profundidad de sondaje, sangrado al sondaje y supuración, evaluando la progresión de la enfermedad en el tiempo. Diversos ensayos clínicos aleatorizados han buscado evaluar los efectos de diferentes antibióticos sistémicos, usando terapias dobles como la combinación de metronidazol y amoxicilina. ${ }^{9}$ Sin embargo, estos antibióticos exigen regímenes de dosificación necesarios que abarcan de dos a cuatro veces al día, durante 5 a 14 días contribuyendo en ocasiones a la disminución de la tasa de éxito debido al incumplimiento de la dosificación. ${ }^{10}$

Por otra parte la azitromicina, antibiótico de la familia de los macrólidos, posee un régimen de dosificación bastante simple y efectos secundarios limitados manteniendo concentraciones más altas y sostenidas en el fluido crevicular gingival que en el suero, además de poseer un amplio espectro de acción antimicrobiana hacia las bacterias anaerobias. ${ }^{11}$

Sayán y col. ${ }^{12}$ en el 2013, realizaron un estudio para evaluar la eficacia de la azitromicina asociada al raspado y alisado radicular en pacientes con enfermedad periodontal crónica, encontrando que el grupo que recibió la terapia periodontal convencional más azitromicina obtuvo una respuesta clínica favorable superior al grupo control, en términos de profundidad al sondaje y nivel de inserción clínica. Estas diferencias fueron mayores durante los primeros meses de evaluación, sin embargo hasta la última evaluación realizada a los 6 meses los parámetros evaluados resultaron no significativos.

Buset SL. y col. ${ }^{13}$ en el 2015, encontraron mediante una revisión sistemática un beneficio potencial de la administración sistémica de azitromicina como adyuvante a la terapia periodontal no quirúrgica en pacientes con periodontitis crónica.

Por su parte Zhang Z. y col. ${ }^{14}$ en el 2015, encontraron un beneficio adicional de la administración sistémica de azitromicina en las bolsas periodontales inicialmente profundas pero no en los sitios de poca profundidad o moderados, así mismo mostró una tendencia a disminuir su efecto en relación al tiempo.

Sin embargo el estudio de Fonseca y col. ${ }^{15}$ en 2015 demostraron mediante un ensayo clínico controlado en 85 pacientes que la administración simultanea del azitromicina al raspado y alisado radicular no provee ningún beneficio significativo independiente del protocolo usado.

Con todo ello, el empleo de estos antimicrobianos no sólo puede traer efectos beneficiosos sino también algunos riesgos que puede ocasionar al sistema microbiano. Al no ser una terapia específica no solo afectaría a patógenos periodontales sino también a otros microorganismos residentes habituales y que más adelante podrían transformarse en resistentes o menos susceptible, comprometiendo la eficacia de la terapia antimicrobiana y el aumento de un fracaso clínico. ${ }^{16}$

El empleo de antimicrobianos sistémicos en el tratamiento de periodontitis demostrados mediante ensayos clínicos aleatorios bien realizados deben conducir a un cambio en los protocolos de tratamiento periodontal, por esta razón se propuso realizar el presente estudio; cuyo propósito fue evaluar la eficacia de la antibióticoterapia sistémica con azitromicina asociada al raspado y alisado radicular para el tratamiento de pacientes con periodontitis crónica.

\section{MATERIAL Y MÉTODO}

El presente estudio fue un ensayo clínico controlado aleatorizado y triple ciego en grupos paralelos. La población estuvo constituida por los pacientes que acudieron con el diagnóstico de periodontitis crónica al servicio de periodoncia de la escuela de odontología en la Clínica Dental de la Universidad Católica Los Ángeles de Chimbote, sede Trujillo, entre marzo y diciembre del 2015. Determinándose una muestra de 30 pacientes divididos en dos grupos, mediante la fórmula que corresponde a comparación de medias.

Los criterios de inclusión para los pacientes con periodontitis crónica fueron: edad entre 30 y 60 años con enfermedad periodontal crónica de magnitud moderada a avanzada. Se consideró como periodontitis moderada a avanzada aquellos sujetos con al menos 15 piezas dentarias y al menos 6 lugares con profundidad de sondaje (distancia en milímetros desde el margen gingival al fondo de la bolsa periodontal) entre 5 y $7 \mathrm{~mm}$ y un nivel de inserción clínica (distancia desde la unión esmalte-cemento hasta el fondo de la bolsa periodontal) entre 5 y $10 \mathrm{~mm}$ antes de iniciar el tratamiento y evidencia radiográfica de destrucción ósea alveolar.

Fueron excluidos en el estudio los pacientes que estuvieron recibiendo terapia con antibióticos, analgésicos o antiinflamatorios durante los 30 días previos a su incorporación al estudio, así como aquellos que referían ser alérgicos a los macrólidos, fumadores, alcohólicos y/o drogadictos o aquellos que presentaron enfermedades sistémicas, tampoco fueron incluidas gestantes o pacientes que presentaron alteraciones físicas y mentales que pudieran impedir el cumplimiento de las indicaciones.

Fueron eliminados aquellos pacientes que no acudieron a sus citas de control en tiempo indicado y los que no cumplieron con las indicaciones posoperatorias.

Para la ejecución de la presente investigación, se contó con la aprobación de la escuela de odontología de la Universidad Católica los Ángeles de Chimbote, considerando los principios de la Declaración de Helsinki y la ley General de salud del Perú (Ley no $26842^{\circ}$ ).

La evaluación y medición clínica fue realizada por el investigador, quien fue calibrado con un especialista mediante una prueba piloto alcanzando un coeficiente de correlación intraclase de $0.924-0.935$ para la evaluación intraoperador y de 0.929 a 1.000 para la evaluación interoperador, demostrando un índice de correlación casi perfecto.

Se seleccionaron de manera no aleatoria por conveniencia en dos grupos. El primero de ellos, denominado grupo A, fue enmascarado para el operador, paciente y estadístico hasta el final de la elaboración de las tablas. Luego de realizado el RAR recibió una cápsula de azitromicina $500 \mathrm{mg}$ de laboratorios Farmindustria Lote N. 11171104, cada 24 horas por 3 días. Cada paciente del grupo de estudio recibió llamadas telefónicas diarias con la finalidad de recordarles el cumplimiento de su dosis.

Mientras que el segundo grupo, denominado grupo B, también fue enmascarado para el operador, paciente y estadístico hasta el final de la elaboración de las tablas. Luego de realizado el RAR recibió una cápsula placebo conteniendo gel de glucosa elaborados con todas las características físicas de la tableta de azitromicina, la cual tomó 1 dosis diaria por 3 días.

Se realizaron controles a los 3, 4 y 5 meses. Evaluando en cada control la profundidad de sondaje periodontal y el nivel de inserción clínica. 
Tabla 1. Eficacia de la azitromicina asociada al raspado y alisado radicular para el tratamiento de pacientes con periodontitis crónica

\begin{tabular}{|c|c|c|c|c|c|c|}
\hline \multicolumn{3}{|c|}{ GRUPO DE ADMINISTRACIÓN } & \multicolumn{3}{|c|}{ TIEMPO } & 5 MESES \\
\hline \multirow{8}{*}{$\begin{array}{l}\text { Medida de profundidad del } \\
\text { sondaje }\end{array}$} & & Media & 5.32 & 2.97 & 2.77 & 2.65 \\
\hline & RAR + AZITROMICINA & $\begin{array}{l}\text { Desviación } \\
\text { estándar }\end{array}$ & 0.23 & 0.47 & 0.45 & 0.35 \\
\hline & & Media & 5.37 & 3.11 & 2.95 & 2.89 \\
\hline & RAR + PLACEBO & $\begin{array}{l}\text { Desviación } \\
\text { estándar }\end{array}$ & 0.31 & 0.54 & 0.36 & 0.44 \\
\hline & \multirow{2}{*}{ Tiempo } & $\mathrm{F}$ & \multicolumn{4}{|c|}{323.32} \\
\hline & & $\mathrm{p}$ & \multicolumn{4}{|c|}{0.000} \\
\hline & \multirow{2}{*}{ Tiempo * Grupo } & $\mathrm{F}$ & \multicolumn{4}{|c|}{0.45} \\
\hline & & $\mathrm{p}$ & \multicolumn{4}{|c|}{0.717} \\
\hline \multirow{8}{*}{ Nivel de inserción clínica } & & Media & 7.32 & 4.85 & 4.54 & 4.43 \\
\hline & RAR + AZITROMICINA & $\begin{array}{l}\text { Desviación } \\
\text { estándar }\end{array}$ & 0.53 & 0.68 & 0.58 & 0.55 \\
\hline & & Media & 7.03 & 4.76 & 4.57 & 4.52 \\
\hline & RAR + PLACEBO & $\begin{array}{l}\text { Desviación } \\
\text { estándar }\end{array}$ & 0.81 & 1.04 & 0.75 & 0.79 \\
\hline & \multirow{2}{*}{ Tiempo } & $\mathrm{F}$ & \multicolumn{4}{|c|}{257.25} \\
\hline & & $\mathrm{p}$ & \multicolumn{4}{|c|}{0.000} \\
\hline & \multirow{2}{*}{ Tiempo * Grupo } & $\mathrm{F}$ & \multicolumn{4}{|c|}{1.15} \\
\hline & & $p$ & \multicolumn{4}{|c|}{0.347} \\
\hline
\end{tabular}

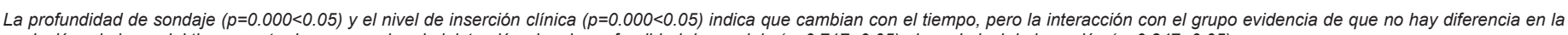
evolución a lo largo del tiempo entre los grupos de administración ni en la profundidad de sondaje $(p=0.717>0.05)$ ni en el nivel de inserción $(p=0.347>0.05)$

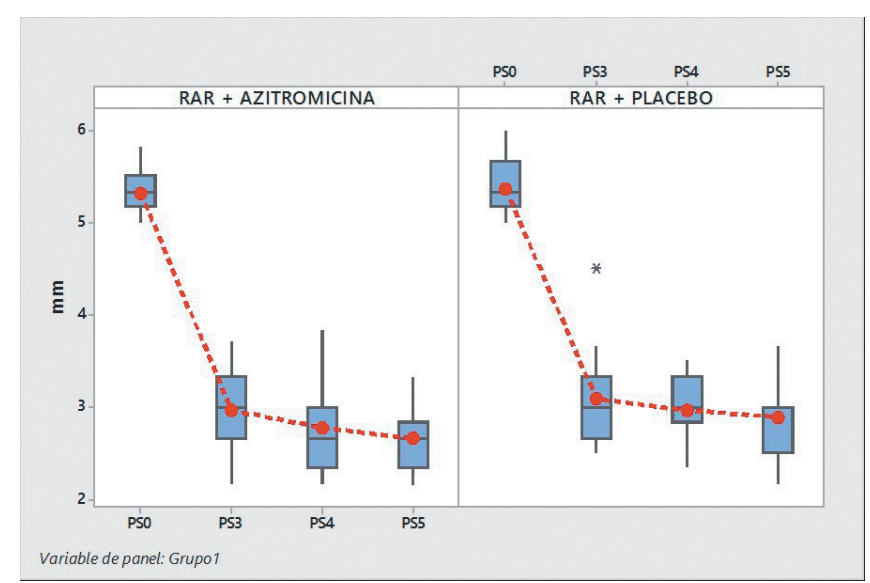

Gráfico 1.

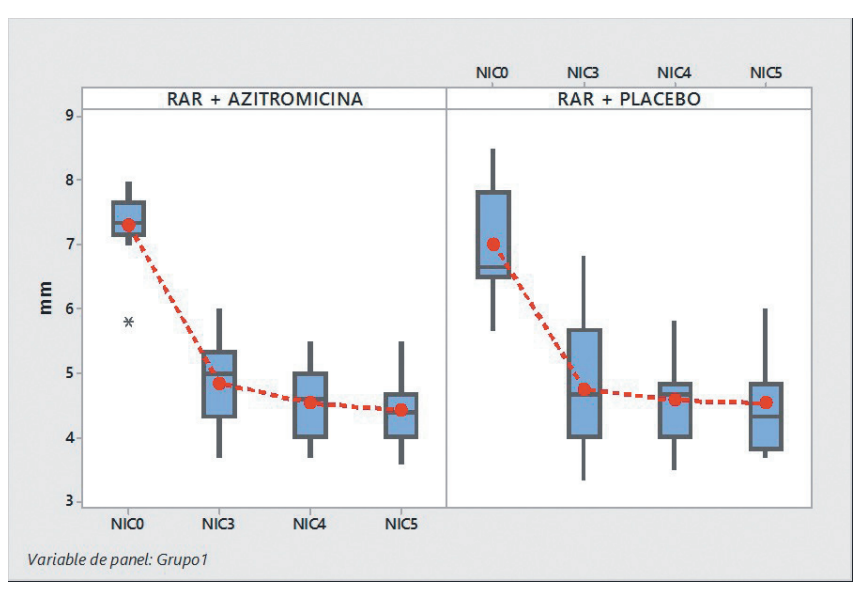

Gráfico 2. 
Ambos grupos recibieron pastas dentales Kolinos Herbal y cepillos dentales Oral B Pro-salud, además de la técnica de higiene de Bass modificada en cada control.

La azitromicina + RAR fue comparada a la administración RAR + placebo, para el tratamiento de pacientes con periodontitis crónica, al basal a los 3, 4 y 5 meses post tratamiento, empleando el test $T$ de Student para comparación de medias. La evaluación de la eficacia de la azitromicina a lo largo del tiempo, comparando los dos tratamientos fue realizada empleando el análisis de medidas repetidas a través del coeficiente Lambda de Wilks, a una significancia estadística considerada de $p<0.05$.

\section{RESULTADOS}

La investigación incluyó a un total de 30 pacientes, de los cuales 15 recibieron azitromicina $500 \mathrm{mg}$ VO como terapia antibiótica y 15 pacientes placebo. Se observó que las medidas de profundidad y nive de inserción clínica mejoraron en ambos grupos (tabla 1), sin embargo al realizar la prueba estadística para la comparación de grupos, estas no mostraron diferencias significativas ( $p=>0.05)$.

La medida de la profundidad al sondaje cambió con el tiempo tanto en el grupo que recibió azitromicina como el grupo que recibió placebo $(p=0.000<0.05)$, pero no se evidencia diferencia en la evolución entre los grupos de administración ( $p=0.717>0.05$ ) (gráfico1)

En cuanto al nivel de inserción clínica también cambió con el tiempo en ambos grupos $(p=0.000<0.05)$, sin embargo no se aprecia diferencia en la evolución al comparar los grupos que recibieron azitromicina y placebo $(p=0.347>0.05)($ grafico 2$)$

\section{DISCUSIÓN}

La azitromicina, es un macrólido bacteriostático cuyo espectro de acción cubre bacterias anaerobias estrictas como los periodontopatógenos. ${ }^{17}$ Presenta buenas propiedades farmacológicas como una rápida absorción, alta concentración tisular y prolongada vida media lo cual permite una sola administración diaria de $500 \mathrm{mg}$ por un periodo de 3 días. ${ }^{11,18}$ Este sencillo protocolo de administración así como su baja incidencia de efectos secundarios tiende a reducir los problemas relacionados con el cumplimiento del paciente, motivo por el cual ha sido considerado por algunas investigaciones para su empleo como terapia coadyuvante en la enfermedad periodontal; así, Oteo y col. $^{18}$ en el 2010, demostraron que la administración conjunta de la azitromicina más el raspado y alisado radicular tenían un efecto clínico superior al grupo control con placebo, ellos evaluaron la profundidad a sondaje así como el nivel de inserción clínica, sin embargo ese efecto superior solo se encontró en los cinco primeros meses, no encontrando diferencias a partir del sexto mes. Resultados similares fueron encontrados por Sayán y col, ${ }^{12}$ Sampaio y col, ${ }^{17}$ Mascarenhas y col, ${ }^{19}$, y Yashima y col. ${ }^{20}$ quienes evaluaron también al mismo antibiótico por el mismo periodo de tiempo, encontrando también solo efecto superior en el grupo que recibió azitromicina hasta el quinto mes.

En el presente estudio se encontró que ambos grupos mostraron una disminución de la profundidad al sondaje y ganancia de inserción clínica, sin embargo no se encontró diferencias estadísticamente significativas entre los grupos de azitromicina y el grupo placebo $(p>0.05)$, resultados que coinciden con los encontrados con Han y col, ${ }^{2}$ Dastoor y col. ${ }^{22}$ y Smith y col. ${ }^{23}$ quienes también evaluaron los mismos parámetros clínicos por un periodo de 6 meses y además realizaron estudios microbiológicos donde se evidenció una disminución de microorganismos periodontales en ambos grupos pero sin mostrar diferencias significativas.

Al realizar la evaluación individual de los parámetros clínicos, tampoco se encontraron diferencias entre el grupo control y de estudio, hallándose similares promedios en las medias de la profundidad al sondaje y nivel de inserción clínica a los tres, cuatro y cinco meses.
Estos resultados difieren con los evaluados por Gomi y col..$^{23}$ quienes encontraron diferencias significativas en el grupo de estudio $(2.4 \pm 0.8$ $\mathrm{mm})$ respecto al grupo control $(3.3 \pm 0.4 \mathrm{~mm})(P<0.05)$ al medir la profundidad al sondaje, sin embargo no observó diferencia en el nivel de inserción clínica, $(4.8 \pm 1.0 \mathrm{~mm}$ y $5.7 \pm 1.0 \mathrm{~mm})(P>0.05)$.

Uno de los trabajos con mayor tiempo de seguimiento fue realizado por Ramberg y col.24 quienes hicieron evaluaciones al año 1, 3, 5 y 13, encontrando solo efecto benéfico superior en el grupo que recibió antibioticoterapia durante el primer año, en las subsiguientes evaluaciones no se encontró diferencias estadísticamente significativas entre los grupos. Se hace importante en este punto señalar, si la administración de antimicrobianos sistémicos solo brinda un efecto inicial, sin encontrar beneficios a mediano y largo plazo, con la consecuente modificación y/o mutación del ecosistema bacteriano del huésped por cada administración que recibe el paciente se debería instaurar protocolos restringiendo su uso solo a casos específicos en donde su empleo está justificado, como el caso de la enfermedad periodontal de avance rápido o de aparición temprana, Los resultados de esta investigación han mostrado en un plazo de seguimiento de 5 meses que no existe evidencia que justifique el empleo de azitromicina como terapia complementaria en pacientes con enfermedad periodontal crónica.

Esta investigación pretendió contribuir a establecer o mejorar el protocolo de atención de los pacientes con enfermedad periodontal crónica. Consideramos que se deben realizar más ensayos clínicos que permitan determinar si pautas habituales de antibióticoterapia son eficaces como coadyuvantes al RAR. Considerar que los antibióticos sistémicos no son los únicos tratamientos complementarios, sino también los agentes tópicos como la Clorhexidina son alternativas viables. Las ventajas o desventajas de cada una de estas estrategias en la práctica clínica, debe ser tomado en cuenta por el odontólogo al obtener evidencia científica y evaluar la mejor opción para cada paciente.

\section{RESPONSABILIDADES ÉTICAS}

Protección de personas y animales. Los autores declaran que los procedimientos seguidos se conformaron a las normas éticas del comité de experimentación humana responsable y de acuerdo con la Asociación Médica Mundial y la Declaración de Helsinki.

Confidencialidad de los datos. Los autores declaran que han seguido los protocolos de su centro de trabajo sobre la publicación de datos de pacientes. Derecho a la privacidad y consentimiento informado. Los autores han obtenido el consentimiento informado de los pacientes y/o sujetos referidos en el artículo. Este documento obra en poder del autor de correspondencia.

\section{LUGAR DONDE SE REALIZÓ EL ESTUDIO} Perú

Universidad Católica los Ángeles de Chimbote (ULADECH). Trujillo-

\section{FINANCIAMIENTO}

El presente estudio fue autofinanciado

\section{CONFLICTO DE INTERESES}

Se declaran no tener ningún conflicto de intereses.

\section{AGRADECIMIENTO}

A los docentes de las asignaturas de Periodoncia I y II de la Clínica Estomatológica de la Universidad Católica Los Ángeles de Chimbote. 


\section{Bibliografía}

1. Socransky SS. Relationship of bacteria to the etiology of periodontal disease. J Dent Res 1970;49:203-222. 2.

2. Han B, Emingil G, Ozdemir G et al. Azithromycin as an adjunctive treatment of generalized severe chronic periodontitis: clinical, microbiologic, and biochemical parameters. J Periodontol 2012:83:1480-149.

3. Watanabe K, Frommel TO. Detection of Porphyromonas gingivalis in ora plaque samples by use of the polymerase chain reaction. J Dent Res 1993: 72 : 1040-1044.

4. Zappa U, Reinking-Zappa M, Graf H, Gmür R, Savitt E. Comparison of serological and DNA probe analyses for detection of suspected periodontal pathogens in subgingival plaque samples. Arch Oral Biol. 1990;35 Suppl:161S-164S.

5. Faveri M, Feres M, Shibli JA, Hayacibara RF, Hayacibara MM, de Figueiredo LC. Microbiota of the dorsum of the tongue after plaque accumulation: an experimental study in humans. J Periodontol 2006: 77: 1539-1546.

6. Faveri M, Figueiredo LC, Duarte PM, Mestnik MJ, Mayer MP, Feres M. Microbiological profile of untreated subjects with localized aggressive periodontitis. $J$ Clin Periodontol 2009: 36: 739-749.

7. Goncalves LFH, Fermiano D, Feres M, Figueiredo LC, Teles FRP, Mayer MPA et al. Levels of Selenomonas species in genereralized aggressive periodontitis. $J$ Periodontal Res 2012: 47: 711-718.

8. Haffajee AD, Bogren A, Hasturk H, Feres M, Lopez NJ, Socransky SS. Subgingival microbiota of chronic periodontitis subjects from different geographic locations. J Clin Periodontol 2004: 31: 996-1002.

9. Herrera D, Sanz M, Jepsen S, Needleman I, Roldan S. A systematic review on the effect of systemic antimicrobials as an adjunct to scaling and root planing in periodontitis patients. J Clin Periodontol 2002;29(suppl 3):136-159.

10. Greenberg RN. Overview of patient compliance with medication dosing: a literatura review. Clin Ther 1984;6:592-599

11. Pérez H. Farmacología Y Terapéutica Odontológica. Editorial Médica. Celsus $2^{a}$ edición. Colombia, 2005.

12. Sayán C, Chumpitaz V. Eficacia de la azitromicina asociada al raspaje y alisado radicular para el tratamiento de pacientes con periodontitis crónica. Tesis para optar el título de Cirujano Dentista. Universidad Mayor de San Marcos. 2013.

13. Buset SL, Zitzmann NU, Weiger R, Walter C. Non-surgical periodontal therapy supplemented with systemically administered azithromycin: a systematic review of RCTs. Clin Oral Investig. 2015 Nov;19(8):1763-75.

14. Zhang Z, Zheng Y, Bian X. Clinical effect of azithromycin as an adjunct to non-surgical treatment of chronic periodontitis: a meta-analysis of randomized controlled clinical trials. J Periodontal Res. 2015 Sep 12.
15. Fonseca DC, Cortelli JR, Cortelli SC, Miranda Cota LO, Machado Costa LC, Moreira Castro MV, et al. Clinical and Microbiologic Evaluation of Scaling and Root Planing per Quadrant and One-Stage Full-Mouth Disinfection Associated With Azithromycin or Chlorhexidine: A Clinical Randomized Controlled Trial. J Periodontol. 2015 Dec;86(12):1340-51.

16. Millones-Gómez P, Huamaní-Munoz W. Efectividad de la antibioticoterapia en la reducción de la frecuencia de alveolitis seca postexodoncia simple. Ensayo clínico aleatorizado de grupos en paralelo, controlado y ciego simple. Rev Esp Cir Oral Maxilofac. 2014. http://dx.doi.org/10.1016/j.maxilo.2014.04.004

17. Armitage G. Periodontal diagnose and classification of periodontal diseases Periodontology 2000, 2004; 34: 9-21.

18. Sampaio E, Rocha M, Figueiredo LC et al. Clinical and microbiological ef fects of azithromycin in the treatment of generalized chronic periodontitis: a randomized placebo-controlled clinical trial. J Clin Periodontol 2011;38:838-846.

19. Oteo A, Herrera D, Figuero E, O'Connor A, Gonzalez I, Sanz M. Azithromycin as an adjunct to scaling and root planing in the treatment of Porphyromonas gingivalis-associated periodontitis: a pilot study. J Clin Periodontol 2010;37:10051015.

20. Mascarenhas P, Gapski R, Al-Shammari K et al. Clinical response of azithromycin as an adjunct to non-surgical periodontal therapy in smokers. J Periodontol 2005;76:426-436

21. Yashima A, Gomi k, Maeda N, Arai T. One-stage full-mouth versus partialmouth scaling and root planning during the effective half-life of systemically administered azithromycin. J Periodontol 2009: 80: 1406-1413.

22. Dastoor SF, Travan S, Neiva RF, Rayburn LA, Giannobile WV, Wang HL. Effect of adjunctive systemic azithromycin with periodontal surgery in the treatmen of chronic periodontitis in smokers: a pilot study. J Periodontol 2007;78:18871896.

23. Smith SR, Foyle DM, Daniels J, Joyston-Bechal S, Smales FC, Sefton A, et al. A double-blind placebocontrolled trial of azithromycin as an adjunct to nonsurgical treatment of periodontitis in adults: clinical results. J Clin Periodonto 2002: 29: 54-61.

24. Gomi K, Yashima A, lino $\mathrm{F}$ et al. Drug concentration in inflamed periodontal tissues after systemically administered azithromycin. J Periodontol 2007;78:918-923

25. Ramberg P, Rosling B, Serino G, Hellström MK, Socransky SS, Lindhe J. The long-term effect of systemic tetracycline used as an adjunct to non-surgical treatment of advanced periodontitis. J Clin Periodontol. 2001 May;28(5):446-52. 